\title{
Clinically significant anxiety as a risk factor for dementia in the elderly community
}

Santabárbara J, Lopez-Anton R, de la Cámara C, Lobo E, Gracia-García P, Villagrasa B, Bueno-Notivol J, Marcos G, Lobo A. Clinically significant anxiety as a risk factor for dementia in the elderly community

Objective: To evaluate whether clinically significant anxiety is an independent risk factor for dementia, taking into account both depression among potentially confounding factors and the competing risk of death.

Method: During the Zaragoza Dementia and Depression (ZARADEMP) study, a random sample of community dwellers aged 55 years or older was assessed $(n=4803)$, and a two-wave, 4.5 -year follow-up was completed. Geriatric Mental State (GMS)-AGECAT criteria were used to diagnose anxiety and DSM-IV criteria were applied to diagnose incident dementia. The multivariate Fine and Gray regression model was implemented to calculate dementia risk.

Results: Compared with non-cases (GMS-AGECAT criteria), the incidence rate of dementia was significantly higher in subcases of anxiety, and particularly significant in the cases of anxiety (incidence rate ratio (IRR): $2.77 ; P=0.010$ ). Cases of anxiety, but not subcases, at baseline were significantly associated with dementia risk (adjusted subdistribution hazard ratio (SHR): $2.7 ; P=0.019$ ).

Conclusion: Clinically significant anxiety is associated with an almost threefold increase in the risk of dementia in the population, even when controlling for depression and considering mortality in the competing risks model.

\author{
J. Santabárbara ${ }^{1,2,3}$, \\ R. Lopez-Anton 2,3,4, \\ C. de la Cámara $2,3,5,6$ \\ E. Lobo ${ }^{1,2,3}$, \\ P. Gracia-García ${ }^{2,3,5,7}$, \\ B. Villagrasa ${ }^{6}, \mathrm{~J}$ Bueno-Notivol ${ }^{1}$, \\ G. Marcos ${ }^{1,2,3}$, A. Lobo ${ }^{2,3,5}$
}

${ }^{1}$ Department of Microbiology, Preventive Medicine and Public Health, Universidad de Zaragoza, Zaragoza, Spain

${ }^{2}$ Instituto de Investigación Sanitaria Aragón, Zaragoza,

Spain, ${ }^{3}$ Centro de Investigación Biomédica en Red de

Salud Mental (CIBERSAM), Ministry of Science and

Innovation, Madrid, Spain, ${ }^{4}$ Department of Psychology and Sociology, Universidad de Zaragoza, Zaragoza,

Spain, ${ }^{5}$ Department of Medicine and Psychiatry,

Universidad de Zaragoza, Zaragoza, Spain, ${ }^{6}$ Psychiatry

Service, Hospital Clínico Universitario, Zaragoza, Spain,

and ${ }^{7}$ Psychiatry Service, Hospital Universitario Miguel

Servet, Zaragoza, Spain

Key words: dementia; anxiety; risk factor; epidemiology; old age

Antonio Lobo, Department of Psychiatry, Universidad de Zaragoza Calle Domingo Miral, s/n, 50009 Zaragoza,

Spain.

E-mail: alobo@unizar.es

Accepted for publication September 3, 2018

\section{Significant outcomes}

- The incidence rate of dementia was almost 3-fold higher in cases of clinically significant anxiety compared with non-cases.

- Clinically significant anxiety confers a 2.7-fold risk of dementia, even when controlling for depression and using a competing risk model that minimizes the probability of overestimating the risk of disease in the presence of high rates of mortality.

- The association between subcases of anxiety at baseline and dementia risk did not reach statistical significance.

\section{Limitations}

- The attrition rate in the study limits the generalizability of results, although this was expected by design.

- Data on ApoE- $\varepsilon 4$ were not obtained.

- We did not control for the use of psychotropic medications, which may be associated with a higher risk of dementia. 


\section{Introduction}

The World Health Organization recognized dementia as a public health priority in 2012 (1). The burden of this condition is expected to increase with societal aging over the coming years (1), even though recent reports show that the agespecific incidence of dementia may be declining (2). In the absence of a disease-modifying treatment or cure, risk reduction of dementia becomes especially relevant. Modifiable risk factors include sedentary lifestyle, diabetes, and cardiovascular disease (3), but other neuropsychiatric symptoms such as depression and anxiety may be important (4). Several population studies have documented an association between depression and dementia, but the nature and extent of this association remains unclear. Two meta-analyses suggest that depression is a true risk factor for dementia $(5,6)$, although other studies suggest that depression is actually a prodromal symptom of diseases that cause dementia (7).

The relationship between anxiety and dementia has received recent attention (8-13), but the nature of this relationship (true association or reverse causation) remains unclear. The few studies of anxiety as a risk factor for dementia have produced conflicting results. Several studies suggest that anxiety is a risk factor for dementia $(8,9,14)$ while three other investigations estimate that dementia risk is increased by $48-70 \%$ in those with anxiety symptoms (11-13). However, de Bruijn et al. (10) found no association of either anxiety symptoms or anxiety disorder. These inconsistencies may reflect variations in factors such as the length of observation, follow-up attrition rate, potentially modifying variables controlled for, or method of assessing either anxiety or dementia. With the exception of de Bruijn et al. (10), who used DSMIV diagnostic criteria to identify anxiety disorder in one sample, others used only scales and questionnaires $(8,10-13)$, and none used methods recommended specifically for use in the elderly (15).

Moreover, previous studies of anxiety and dementia did not consider the competing risk of death, which should probably be mandatory in risk model estimates, particularly in the elderly, as death may preclude the occurrence of dementia (16).

\section{Aims of the study}

We evaluated whether clinically significant anxiety is a risk factor for dementia in a community sample, taking into account the competing risk of death.

\section{Methods}

Sample

Data were obtained from the Zaragoza Dementia and Depression (ZARADEMP) study, a 5-wave, longitudinal community study carried out in Zaragoza, Spain (17). We present data from baseline (Wave I, starting in 1994) and from two follow-up visits (Wave II, starting in 1997; and Wave III, starting in 1999). Briefly, in 1991 a random sample of community-dwelling persons aged 55 years or older was drawn proportionally by age and sex from the census list of Zaragoza (Spain). Institutionalized individuals were included. A total of 4803 subjects underwent at baseline interview (Wave I). Because we were interested in dementia-free individuals, 746 subjects identified as dementia cases or subcases (i.e., those with suggestive but ambiguous symptoms) were not studied further, leaving a residual sample of 4057 for follow-up studies.

The principles of the Declaration of Helsinki were followed throughout. The Ethics Committee of the University of Zaragoza, and the Fondo de Investigación Sanitaria (FIS) approved the study protocol, according to Spanish Law. All individuals included in the study provided written informed consent.

\section{Procedure}

A two-phase screening procedure was used at baseline and in each subsequent wave. First (Phase I), well-trained and regularly supervised lay interviewers conducted the 25- to 90-min ZARADEMP interview at the subjects' home or place of residence. The ZARADEMP interview incorporates validated Spanish versions of several international instruments. The Mini-Mental State Examination (MMSE) $(18,19)$ screens cognitive function. The Geriatric Mental State (GMS), a semistructured standardized clinical interview, assesses the mental state of elderly persons $(20,21)$, yielding data suitable for the AGECAT computer system that identifies psychiatric diagnoses (22). We also used the History and Aetiology Schedule (HAS), a standardized method of collecting history data from a caregiver or directly from reliable respondents (23). The Katz Index $(24,25)$ and the Lawton and Brody Scale $(26,27)$ assessed basic and instrumental activities of daily living. A series of questions regarding medical and psychiatric history from the EURODEM (European Community Concerted Action on the Epidemiology and Prevention of Dementia) Study Risk Factors Questionnaire was also presented (28).

Periodic re-training of interviewers was used to avoid a decay in reliability. In phase II, trained 
supervising research psychiatrists reassessed those individuals considered to be 'probable psychiatric cases', and/or participants with information considered to be unreliable. These interviews were also conducted in the participants' place of residence, and the same instruments were used. Our previous studies support the validity of this casefinding procedure (20).

\section{Dementia assessment and diagnosis}

Participants in the follow-up waves (Waves II and III) were classified in phase I as 'probable cases' of dementia based on the GMS threshold 'global' score $(>1)$ and/or MMSE standard cut-off points $(<24)$. Those with a GMS 'global score' of 1 were considered to be 'sub-cases'. In phase II, all probable cases of dementia were reassessed in their place of residence by a research psychiatrist using the same methods as well as the Hachinski's scale (29) and a brief, previously standardized neurologic examination. Incident dementia was initially diagnosed by the research psychiatrist doing the assessment, but the final DSM-IV diagnosis, made by consensus, required the agreement of at least three psychiatrists in a four-member panel. Previous studies support the validity of this diagnostic process (20). Moreover, to document the accuracy of the panel, a proportion of cases were invited to undergo a hospital-based diagnostic work-up, which incorporated a neuropsychological diagnostic battery and neuroimaging studies. Agreement on the diagnosis of dementia was reached in $95.8 \%$ of the cases.

\section{Anxiety assessment and diagnosis}

The staged GMS-AGECAT approach was applied to diagnose anxiety at baseline. Although the GMS does not map precisely onto the DSM or ICD categories, it was calibrated to detect disorders of clinical severity, and has been used in several studies to describe anxiety disorders (30, 31). This diagnostic approach is valid in community samples, and participants with AGECAT scores of 3,4 , and 5 in the $0-5$ scale are considered to be likely 'cases' of anxiety (clinically significant anxiety requiring clinical intervention); those with scores of 1 and 2 are considered to be 'sub-cases' (mild anxiety), and those with a score of 0 are considered to be 'non-cases' (unaffected) (32).

\section{Covariates}

Potentially confounding factors were assessed at baseline, and included sociodemographic characteristics (age, sex, educational level, marital status, and living alone), medical risk factors (vascular disease, hypertension, and diabetes), health status, depression, and cognitive status.

Education was categorized into three levels: 'illiterate' (unable to read and write, and/or $<2$ years of formal education), 'primary' (complete or incomplete), and 'secondary school or higher'. Marital status was categorized as follows: 'single', 'married or living as a couple', 'divorced or separated', and 'widowed'. Health status was rated according to the HAS criteria and dichotomized as 'good (physical illness absent)' or 'not good (physical illness present)'.

Blood pressure (BP) was measured during the interview using a standard manual tensiometer, using the mean of two BP readings; hypertension was defined as $\mathrm{BP}>140 / 90 \mathrm{mmHg}$ or receiving treatment for hypertension. The presence of vascular risk factors and diabetes was based on the medical history obtained by using the EURODEM Risk Factors Questionnaire (28). The presence of vascular diseases was dichotomized, distinguishing between vascular disease (angina, myocardial infarct, and/or stroke) and no history of vascular disease. Diabetes was dichotomized into persons with a previous medical diagnosis or receiving treatment for diabetes and the absence of diabetes. The diagnosis of depression was based on the AGECAT computer system (33).

\section{Statistical analysis}

Differences between baseline characteristics according to anxiety status were assessed using two-tailed chi-square tests on categorical data, and differences in variables with approximately normal distributions were assessed using analysis of variance.

Standard procedures were used to calculate incidence rate and incidence rate ratio (IRR). The follow-up period ended at the second follow-up examination (Wave III) for the non-demented individuals, at the date of invitation for refusals, at the date of moving away or death (based on actual data from the Civil Registry, 'Padrón Municipal de Habitantes de Zaragoza'), or at the time of onset of dementia for cases. The time of onset of dementia was estimated to be the time from baseline to the midpoint between diagnosis and the previous examination.

We used a multivariate survival analysis with age as a time-scale with delayed entry (34) to study the specific hypothesis that anxiety disorder is associated with an increased risk of overall dementia. In a first step of the survival analysis, 


\section{Santabárbara et al.}

we built the cumulative incidence functions (CIF) (35) for the anxiety disorder groups to estimate the probability of incident overall dementia taking into account the competing event (death) as time progressed (36-38). We tested for equality of CIF across groups (39). Then, to estimate the effect of baseline predictors on the cumulative incidence function, we used the Fine and Gray (36) regression model to estimate the subdistribution hazard. This model modifies the Cox proportional hazards model, allowing for competing risks (death). To explore mechanisms explaining the association, we used two models in which we gradually controlled for potential confounders and/or effects modifiers. Interactions between covariates (sociodemographic characteristics, medical risk factors, health status, depression, and cognitive status) and anxiety diagnosis were assessed before calculating the multivariate regression model, and no statistically significant interactions were detected. To examine the assumption of proportional distribution hazards, we tested the time-varying effect of each covariate using the Scheike and Zhang test (40). Statistical analyses were conducted using $R$ software (http:// www.r-project.org), with the epiR package to analyze epidemiologic data, and the cmprsk and timereg packages for survival analyses.

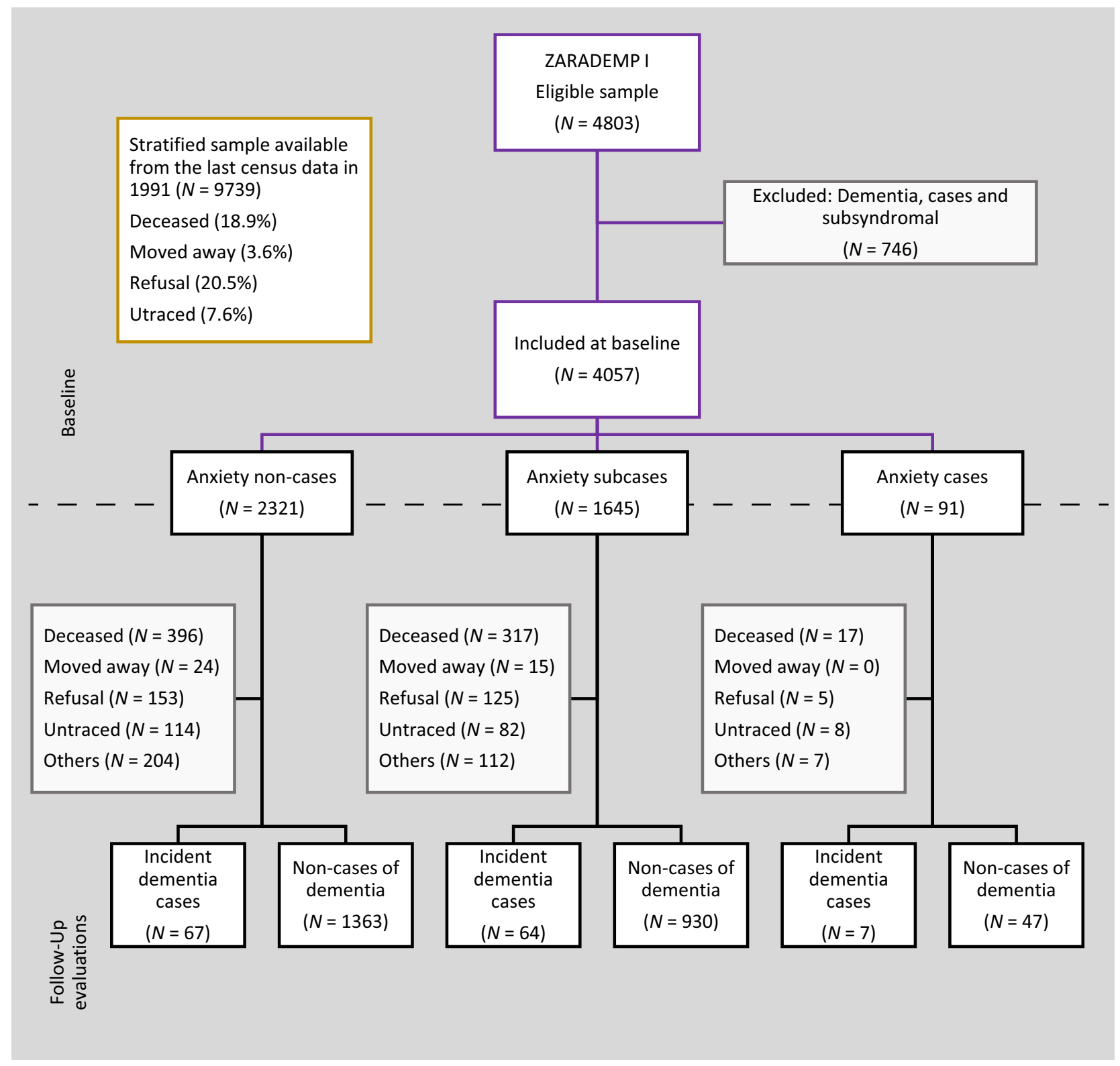

Fig. 1. Study flowchart. AGECAT, Automated Geriatric Examination for computer asisted taxonomy; ZARADEMP, Zaragoza Dementia and Depression Project. 
Anxiety as a risk factor for dementia

\section{Results}

Figure 1 illustrates the flow diagram of the ZARADEMP Project. The refusal rate for initial examination was $20.5 \%$, and 4803 individuals were ultimately interviewed at baseline in 1994. Wave II was completed in 1997, and wave III in 1999. Between the wave I baseline and wave III, 730 $(17.9 \%)$ individuals died, and $849(20.9 \%)$ were lost during follow-up, leaving an examined sample of $2479(61.1 \%)$ for wave III follow-up.

Those lost during follow-up were on average 4.3 years older $(P<0.001)$ and more likely to be illiterate than those re-evaluated; the MMSE scores were also lower among those lost $(P<0.001)$. The lost and examined groups had a similar sex distribution. At baseline, 91 participants $(2.2 \%)$ were diagnosed with anxiety, and $1645(40.5 \%)$ were classified as subcases of anxiety.

The baseline characteristics according to anxiety status are displayed in Table 1. Compared with non-cases, subcases of anxiety were more likely to be female, illiterate, and to live alone; to perform worse cognitively, and to have depression, functional disabilities, and to be unhealthy with diabetes and vascular disease. Compared with non-cases, those who received anxiety diagnoses were more likely to be female, to live alone, to perform worse cognitively, to have depression and disabilities for instrumental ADLs, and to be unhealthy. Compared with subcases, cases of anxiety were more likely to be female, to have depression and disabilities for instrumental ADLs, and to be unhealthy.

The median follow-up time was 4.4 years (interquartile range: 3.0-4.9). At wave III, 138 $(3.4 \%)$ participants were diagnosed as incident cases of dementia (Fig. 1). Seven out of the 91 cases with clinically significant anxiety followed up (amounting a total of 346 person-years follow-up) developed dementia.

Table 2 shows the risk of overall dementia in relation to the anxiety status. Compared with noncases, both the proportion of incident cases and the incidence rate of dementia were higher among subcases, and particularly higher among cases of anxiety, the IRR being significantly higher among the latter $(\mathrm{IRR}=2.77 ; P=0.010)$.

The incidence rate of dementia was higher among cases of anxiety than in subcases, but this differences did not reach statistical significance $(\mathrm{IRR}=1.98 ; P=0.075)$.

Then, when the competing risk of mortality is taken into account in the dementia risk calculation, crude comparison of the CIF by anxiety
Table 1. Baseline characteristics according to anxiety status

\begin{tabular}{|c|c|c|c|c|}
\hline & $\begin{array}{c}\text { Anxiety } \\
\text { non-cases } \\
(N=2321)\end{array}$ & $\begin{array}{c}\text { Anxiety } \\
\text { subcases } \\
(N=1645)\end{array}$ & $\begin{array}{c}\text { Anxiety cases } \\
(N=91)\end{array}$ & $P$ \\
\hline & Mean (SD) & Mean (SD) & Mean (SD) & \\
\hline Age (years) & $72.0(9.1)$ & $72.2(9.2)$ & $72.3(8.6)$ & 0.538 \\
\hline MMSE & $\begin{array}{c}27.4(2.6) \\
N(\%)\end{array}$ & $\begin{array}{c}27.0(2.4)^{*} \\
N(\%)\end{array}$ & $\begin{array}{c}26.4(3.9) \dagger \\
N(\%)\end{array}$ & $<0.001$ \\
\hline Female sex & $1063(48.8 \%)$ & $1096(66.6 \%)^{*}$ & $70(76.9 \%) \dagger+$ & $<0.001$ \\
\hline Education & & & & 0.001 \\
\hline $\begin{array}{c}\text { Illiterate } \\
\text { Primary } \\
\text { school }\end{array}$ & $\begin{array}{c}186(8.0 \%) \\
1688(72.7 \%)\end{array}$ & $\begin{array}{c}152(9.2 \%) \\
1263(76.7 \%)\end{array}$ & $\begin{array}{l}10(11.0 \%) \\
68(74.7 \%)\end{array}$ & \\
\hline $\begin{array}{l}\text { Secondary } \\
\text { school or } \\
\text { higher }\end{array}$ & $447(19.2 \%)$ & $230(13.9 \%)^{*}$ & $13(14.3 \%)$ & \\
\hline Civil status & & & & 0.065 \\
\hline $\begin{array}{c}\text { Married/ } \\
\text { couple }\end{array}$ & 1491 (64.4\%) & 981 (59.8\%) & 56 (61.5\%) & \\
\hline $\begin{array}{l}\text { Divorced/ } \\
\text { separated }\end{array}$ & $28(1.2 \%)$ & $28(1.7 \%)$ & $2(2.2 \%)$ & \\
\hline Widowed & 586 (25.3\%) & $481(29.3 \%)$ & $27(29.7 \%)$ & \\
\hline Living alone & $371(16.0 \%)$ & $306(18.6 \%)^{*}$ & $24(26.4 \%) \dagger$ & 0.007 \\
\hline Depression & 68 (2.9\%) & $342(20.8 \%)^{*}$ & $45(49.5 \%) \dagger$ & $<0.001$ \\
\hline \multicolumn{5}{|l|}{ Disability } \\
\hline $\begin{array}{l}\text { Instrumental } \\
\text { ADLs }\end{array}$ & $245(10.6 \%)$ & $241(14.7 \%)^{*}$ & $26(28.9 \%)+\div$ & $<0.001$ \\
\hline Basic ADLs & 117 (5.1\%) & $155(9.4 \%)^{*}$ & $5(5.5 \%)$ & $<0.001$ \\
\hline $\begin{array}{l}\text { Health status } \\
\text { ('not good') }\end{array}$ & $1084(46.7 \%)$ & $952(58.0 \%)^{*}$ & $65(71.4 \%) \dagger$ & $<0.001$ \\
\hline \multicolumn{5}{|c|}{ Vascular risk factors } \\
\hline Hypertension & 1565 (67.5\%) & 1121 (68.3\%) & 61 (67.0\%) & 0.871 \\
\hline Diabetes & 264 (11.4\%) & $223(13.7 \%)^{*}$ & 14 (15.4\%) & 0.070 \\
\hline $\begin{array}{l}\text { Vascular } \\
\text { disease }\end{array}$ & $236(10.2 \%)$ & $211(12.8 \%)^{*}$ & $12(13.2 \%)$ & 0.030 \\
\hline
\end{tabular}

Data are given as mean (standard deviation) or number (\%). ADLs, activities of daily living; MMSE, Mini-Mental State Examination.

*Significant differences $(P<0.05)$ between 'anxiety subcases' and 'anxiety noncases' in either $t(\mathrm{df}=3964)$ test or $\chi^{2}(\mathrm{df}=1)$ test.

$\dagger$ Significant differences $(P<0.05)$ between 'anxiety cases' and 'anxiety non-cases' in either $t(\mathrm{df}=2410)$ test or $\chi^{2}(\mathrm{df}=1)$ test.

$\$$ Significant differences $(P<0.05)$ between 'anxiety cases' and 'anxiety subcases' in either $t(\mathrm{df}=1734)$ test or $\chi^{2}(\mathrm{df}=1)$ test.

status shows that in the 4.5-year follow-up, compared with the non-cases, the probability of developing dementia was higher in individuals with anxiety, particularly in the anxiety cases (Fig. 2). For example, for individuals aged 85 years, the probability (in percentage) of dementia in the noncases group was $4.0 \%$ (95\% confidence interval [CI]: $2.7 \%-5.7 \%$ ), significantly lower than in both the subcases group $(6.1 \%$; $95 \% \mathrm{CI}: 4.3 \%-8.5 \%)$ and the cases group $(8.7 \%$; $95 \%$ CI: $2.6 \%-$ $19.6 \%$ ).

Table 3 shows the results of the competing risk regression analysis of incident dementia associated with anxiety status. The univariate analysis revealed a significant association between anxiety cases at baseline and dementia risk, and this association persisted in the fully adjusted model 


\section{Santabárbara et al.}

with the inclusion of all potential confounding factors (Subdistribution Hazard Ratio $[\mathrm{SHR}]=2.7$; $P=0.019)$. No significant association between subcases of anxiety at baseline and dementia risk was found in either model.

\section{Discussion}

This study shows that, compared with non-cases, clinically significant anxiety increases the risk of dementia almost three times, although the difference did not reach statistical significance in the anxiety subcases. While some, but not all, of the previous reports showing an association of anxiety with incident dementia (8), also controlled for depression, this is the first study in which a competing risk model was used (41). This is an advantage over traditional models (e.g., Kaplan-Meier and Cox regression), which do not take into account competing risks of death and may overestimate the risk of disease in the presence of high rates of mortality. This is particularly important in studies of the elderly (16). In support of this method, a secondary analysis in this study demonstrated that, according to the Kochar-Lam-Yip test (42), the risk of death was significantly higher than the risk of overall dementia $(P<0.001$, data not shown $)$. Moreover, in controlling for the effect of age at baseline in the risk of dementia, we, like Petkus et al. (11), used exact age as the time-scale, in contrast to previous studies using a time-onstudy and including age as a covariate in the regression models $(10,12,13)$. The exact age as time-scale is preferred for avoiding a bias on effect estimates in samples of older adults because age is strongly associated with some covariates (e.g., chronic diseases) (34). We have previously documented in this same sample that the dementia risk increases significantly with an individual's age (43).

Most recent reports documenting an association between anxiety and all-cause dementia (8, 9,

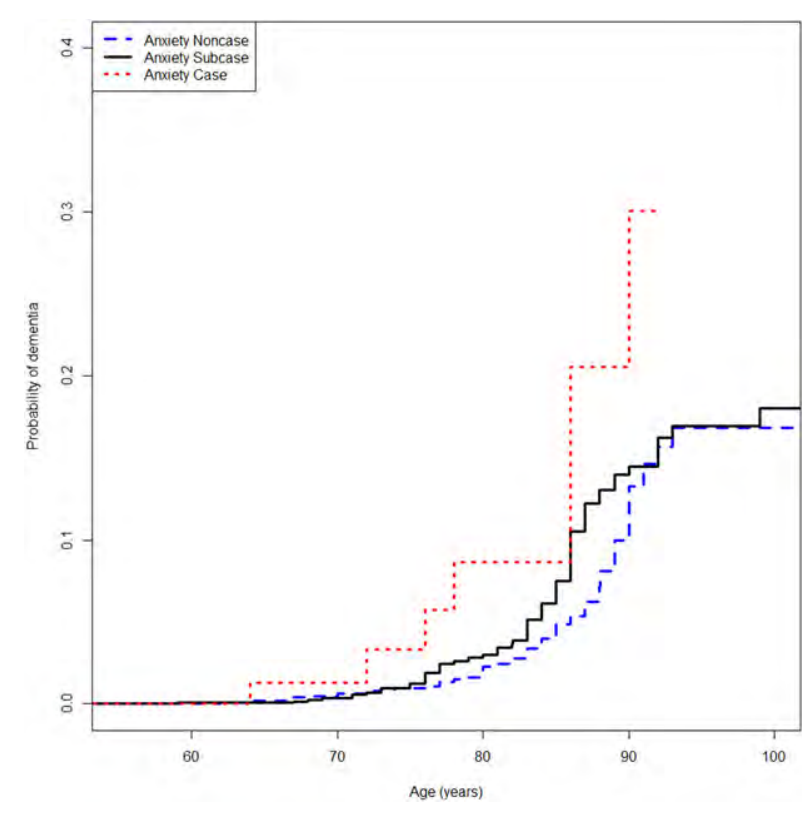

Fig. 2. Probability of incident dementia by anxiety status at baseline in the study sample.

11-13), such as the ones included in the systematic review and meta-analysis of Gulpers et al. (14), used questionnaires and scales in the assessment of anxiety. However, Therrien and Hunsley (15) concluded that, contrary to the GMS used in our study, most of these instruments lack sufficient psychometric evidence to warrant their use with older adults. Moreover, in contrast to those questionnaires documenting anxiety symptoms, the GMS-AGECAT we have used has been shown to detect in the community the 'cases' considered to requery clinical intervention $(31,32)$. De Bruijn et al. did use DSM-IV criteria to assess anxiety (10), but reported that neither anxiety disorder nor anxiety symptoms were associated with an increased risk of dementia. Nonetheless, they did not control for the competing risk of death and considered that the 'generalizability of results to other populations was limited' because of the special characteristics of their sample.

Table 2. Incident cases and incidence rates of overall dementia according to anxiety status at baseline

\begin{tabular}{|c|c|c|c|c|c|}
\hline & Cases, $n(\%)$ & Person-years & Incidence Rate (95\% CI) & $\operatorname{IRR}(95 \% \mathrm{CI})^{*}$ & $P$ \\
\hline \multicolumn{6}{|l|}{ Anxiety status at baseline } \\
\hline Non-cases $(n=2321)$ & $67(2.9 \%)$ & 9199.96 & 7.28 (5.73-9.25) & & \\
\hline Subcases $(n=1645)$ & $64(3.9 \%)$ & 6467.61 & 9.90 (7.75-12.64) & $1.36(0.96-1.91)$ & 0.079 \\
\hline Cases $(n=91)$ & $7(7.7 \%)$ & 346.47 & 20.20 (9.63-42.38) & $2.77(1.27-6.04)$ & 0.010 \\
\hline
\end{tabular}

Cl, Confidence Interval; IRR, Incidence Rate Ratio; ZARADEMP, Zaragoza Dementia and Depression Project (at 4.5-year follow-up). Bold entries indicate statistically significant values.

${ }^{*}$ Reported IRR of general dementia are related to non-case of anxiety; 95\% confidence intervals are shown for both incidence rate and IRR; $P$ values related to IRR were from the $z$ test. 
Table 3. Risk of overall dementia for Fine and Gray models according to anxiety status at baseline

\begin{tabular}{|c|c|c|c|c|}
\hline & \multicolumn{2}{|c|}{ Gender-adjusted model } & \multicolumn{2}{|c|}{ Multivariate model } \\
\hline & SHR $(95 \%$ Cl)* & $P$ & SHR $(95 \%$ Cl)* & $P$ \\
\hline \multicolumn{5}{|c|}{ Anxiety status at baseline } \\
\hline Non-cases & 1 & - & 1 & - \\
\hline Subcases & $1.21(0.86-1.70)$ & 0.270 & $1.32(0.91-1.89)$ & 0.140 \\
\hline Cases & $2.51(1.16-5.45)$ & 0.020 & $2.74(1.18-6.35)$ & 0.019 \\
\hline
\end{tabular}

SHR, Subdistribution Hazard ratio. Bold entries mean the SHR is statistically significant.

${ }^{*}$ Reported SHR of dementia is related to non-cases, Cls and $P$ values related to SHR were from "normal approximation" of Wald $\chi^{2}$ test with $1 \mathrm{df}$. Gender-adjusted Model included anxiety status and sex. Multivariate Model included terms for gender-adjusted model plus sociodemographic characteristics (educational level, marital status and living alone), medical risk factors (vascular disease, hypertension and diabetes), health status, depression and cognitive status at baseline (MMSE score). Both models used age as time-scale.

It might be argued that the association found between anxiety and incident cases of dementia is the effect of preclinical disease (i.e., reverse causation) or prodromal changes rather than a risk factor for dementia $(14,44)$. Nevertheless, our findings support the notion of increased risk, because contrary to studies such as de Bruijn et al. (10), Petkus et al. (11), and Acosta et al. (13), we excluded at baseline all subcases of dementia (AGECAT criteria) to minimize the possibility of including in the cohort individuals with mild cognitive deficits. Moreover, only cases of anxiety and not subcases in our study were associated with incident dementia. If anxiety was prodromic (45), it would be expected that anxiety subcases would also develop dementia in a 4.5-year period. Additionally, in trying to infer causation, we considered that at least some of the anxiety might relate to early perceived memory difficulties. Therefore, in a post hoc analysis we introduced in the multivariate models the question of subjective memory impairment (GMS criteria), but the results were not substantially altered (anxiety case, $\mathrm{SHR}=2.45, P=0.045$; anxiety subcase, $\mathrm{SHR}=1$. 26 , non-signifcant).

Several hypotheses may explain the increased risk of dementia associated with anxiety disorder. First, anxiety might promote negative neuroplasticity, as suggested by Vance et al. (46), therefore decreasing 'cognitive reserve'. Second, anxiety may induce an accelerated aging across multiple biological processes, as suggested in a recent review (47). Third, there is growing evidence in the literature for the role of central nervous system inflammation in the pathology of the most common dementia in the community, Alzheimer Disease (48), and inflammatory changes have also been shown to be associated with anxiety (49). Therefore, the possibility that these inflammatory changes negatively influence the pathologic processes of Alzheimer Disease should also be considered.

Our study has other strengths, such as the use of a representative population sample, including institutionalized individuals (50). Moreover, we have used instruments validated within the study. The agreement between psychiatrists and AGECAT in making diagnostic decisions was high in several studies and different populations $(22,51)$ and, we controlled for depression, in view of its frequent association with anxiety in the community (52).

Some limitations must be noted, such as the significant attrition from sampling to enrollment. However, the attrition rate was expected by design (17) and we previously argued that our investigation is comparable to several other two-stage epidemiologic studies (17). While we previously documented that the screening method used to detect dementia is particularly sensitive in this population (20), some 'true' cases may have been missed. Because we do not know if the association between anxiety and subsequent discovery of cases is the same for these persons as the association of anxiety with true incident dementia, this limitation is unavoidable. Moreover, the agreement on diagnosis of dementia between panel assessment and hospital diagnosis for cases was satisfactory, but we have no data on agreement for non-cases. It is also apparent that some individuals with incident dementia may have died before they could be examined at follow-up, and therefore we do not know their anxiety status at baseline. In view of reports documenting an increased mortality among the elderly with anxiety (53), it might be expected that death would preclude the development of dementia in a higher proportion of the anxiety cases than in the non-cases. Had this not happened, there would be more incident cases of dementia among the anxiety cases. In such circumstances, the conclusions of this study would be reinforced. Lastly, this study, similar to the studies reviewed in this report, did not control for the use of benzodiazepines, antidepressants, or other psychotropic medications, which some studies have associated with a higher risk of dementia (54-56).

In conclusion, clinically significant anxiety increased the risk of dementia in these study subjects, even when considering mortality in the competing risks model and including depression among the confounding factors to control. These findings may stimulate additional studies of the effect of treating anxiety to decrease the risk of dementia. 


\section{Santabárbara et al.}

\section{Acknowledgements}

The authors acknowledge the contribution of the lay interviewers, senior medical students, and members of the ZARADEMP Workgroup who participated in the study.

\section{Funding}

Supported by Grants from the Fondo de Investigación Sanitaria, Instituto de Salud Carlos III, Spanish Ministry of Economy and Competitiveness, Madrid, Spain (grants 94/1562, 97/1321E, 98/0103, 01/0255, 03/0815, 06/0617, G03/128) and from the Fondo Europeo de Desarrollo Regional (FEDER) of the European Union and Gobierno de Aragón (grant B15_17R).

\section{Declaration of interests}

We declare that C. De-la-Cámara received financial support to attend scientific meetings from Janssen-Cilag, Almirall, Eli Lilly, Lundbeck, Rovi, Esteve, Novartis, and Astrazeneca. Dr. P. Gracia-García received Grant support from Janssen, AstraZeneca, and the Ilustre Colegio de Médicos de Zaragoza; she has received Honorarium from AstraZeneca and Lilly; and she has received travel support from Lilly, Almirall, Lundbeck, Rovi, Pfizer, and Janssen. J.M. None of these activities was related to the current project. The remaining authors have no declaration of interests.

\section{References}

1. World Health Organization. Dementia: a public health priority. Geneva: World Health Organization; 2012. Available at: http://www.who.int/mental_health/publi cations/dementia_report_2012/en/[accessed 7 January 2018].

2. Wu YT, Beiser AS, Breteler MMB et al. The changing prevalence and incidence of dementia over time - current evidence. Nat Rev Neurol 2017;13:327-339.

3. Baumgart M, Snyder HM, Carrillo MC, Fazio S, Kim $\mathrm{H}$, Johns H. Summary of the evidence on modifiable risk factors for cognitive decline and dementia: a population-based perspective. Alzheimers Dement 2015;11:718-726.

4. Prince M, Jackson J, editors. World Alzheimer Report 2014. London: Alzheimer's Disease International; 2014. Available at: https://www.alz.co.uk/research/WorldAlzhe imerReport2014.pdf [accessed 7 January 2018]

5. Diniz BS, Butters MA, Albert SM, Dew MA, Reynolds CF 3rd. Late-life depression and risk of vascular dementia and Alzheimer's disease: systematic review and meta-analysis of community-based cohort studies. $\mathrm{Br} \mathrm{J}$ Psychiatry 2013;202:329-335.

6. Cherbuin N, Kim S, Anstey KJ. Dementia risk estimates associated with measures of depression: a systematic review and meta-analysis. BMJ Open 2015;5:e008853.

7. Singh-Manoux A, Dugravot A, Fournier A et al. Trajectories of depressive symptoms before diagnosis of dementia: a 28year follow-up study. JAMA Psychiatry 2017;74:712-718.

8. Gallacher J, Bayer A, Fish M et al. Does anxiety affect risk of dementia? Findings from the Caerphilly Prospective Study. Psychosom Med 2009;7:659-666.

9. Burton C, Campbell P, Jordan K, Strauss V, Mallen C. The association of anxiety and depression with future dementia diagnosis: a case-control study in primary care. Fam Pract 2013;30:25-30.
10. de Bruisn RF, Direk N, Mirza SS et al. Anxiety is not associated with the risk of dementia or cognitive decline: The Rotterdam Study. Am J Geriatr Psychiatry 2014;22:1382-1390.

11. Petkus AJ, Reynolds CA, Wetherell Jl, Kremen WS, Pedersen NL, Gatz M. Anxiety is associated with increased risk of dementia in older Swedish twins. Alzheimers Dement 2016;12:399-406.

12. Kassem AM, Ganguli M, YafFe $\mathrm{K}$ et al. Anxiety symptoms and risk of dementia and mild cognitive impairment in the oldest old women. Aging Ment Health 2018;22:474-482.

13. Acosta I, Borges G, Aguirre-Hernandez R, Sosa Al, Prince M; 10/66 Dementia Research Group. Neuropsychiatric symptoms as risk factors of dementia in a Mexican population: a 10/66 Dementia Research Group study. Alzheimers Dement 2018;14:271-279.

14. Gulpers B, Ramakers I, Hamel R, Köhler S, Oude Voshaar R, Verhey F. Anxiety as a predictor for cognitive decline and dementia: a systematic review and meta-analysis. Am J Geriatr Psychiatry 2016;24:823-842.

15. Therrien Z, Hunsley J. Assessment of anxiety in older adults: a systematic review of commonly used measures. Aging Ment Health 2012;16:1-16.

16. Berry SD, Ngo L, Samelson EJ, Kiel DP. Competing risk of death: an important consideration in studies of older adults. J Am Geriatr Soc 2010;58:783-787.

17. Lobo A, SAZ P, Marcos G et al. The ZARADEMP Project on the incidence, prevalence and risk factors of dementia (and depression) in the elderly community: II. Methods and first results. Eur J Psychiatry 2005;19:40-54.

18. Folstein MF, Folstein SE, McHugh PR. "Mini-mental state". A practical method for grading the cognitive state of patients for the clinician. J Psychiatr Res 1975;12:189-198.

19. Lово A, SAz P, Marcos G et al. Revalidation and standardization of the cognition mini-exam (first Spanish version of the Mini-Mental Status Examination) in the general geriatric population. Med Clin (Barc) 1999;112:767-774.

20. Lobo A, Saz P, Marcos G, Día JL, De-La-cámara C. The prevalence of dementia and depression in the elderly community in a southern European population. The Zaragoza Study. Arch Gen Psychiatry 1995;52: 497-506.

21. Dewey Me, Copeland JRM, Lobo A, Saz P, Dia JL. Computerized diagnosis from a standardized history schedule: a preliminary communication about the organic section of the HAS-AGECAT system. Int $\mathbf{J}$ Geriatr Psychiatry 1992;7:443-446.

22. Copeland JRM, Dewey ME, Griffiths-Jones HM. A computerized psychiatric diagnosis system and case nomenclature for elderly subjects: GMS and AGECAT. Psychol Med 1986;16:89-99.

23. Dewey ME, Copeland JRM. Diagnosis of dementia from the history and aetiology schedule. Int $\mathrm{J}$ Geriatr Psychiatry 2001;16:912-917.

24. Katz S, Ford AB, Moskowitz RW, Jackson BA, Jaffe MW. Studies of illness in the aged. The index of ADL: a standardized measure of biological and psychosocial function. JAMA 1963;185:914-919.

25. Alvarez Solar M, De Alaiz Rojo A, Brun Gurpegui E et al. Functional capacity of patients over 65 according to the Katz index. Reliability of the method. Aten Primaria 1992;10:812-8816.

26. Lawton MP, Brody EM. Assessment of older people: selfmaintaining and instrumental activities of daily living. Gerontologist 1969;9:179-186. 
27. TÁrRaga L. Evaluación del deterioro cognitivo y funcional de la demencia. Escalas de mayor interés en la Atención Primaria. In: Boada M, TÁrraga L, editors. El Médico Ante la Demencia y su Entorno, Módulo 1. Barcelona: Bayer SA; 1995.

28. LAUNER LJ. European studies on the incidence of dementing diseases. EURODEM Incidence Conferences. Bordeaux, France, 1989 and Cambridge, UK, 1990. Neuroepidemiology 1992;11:1-122.

29. Hachinski VC, Lassen NA, Marshall J. Multi-infarct dementia a cause of mental deterioration in the elderly. Lancet 1974;304:207-209.

30. Kvaal K, McDougall FA, Brayne C, Matthews Fe, Dewey ME. Co-occurrence of anxiety and depressive disorders in a community sample of older people: results from the MRC CFAS (Medical Research Council Cognitive Function and Ageing Study). Int J Geriatr Psychiatry 2008;23:229-237.

31. Prina AM, Ferri CP, Guerra M, Brayne C, Prince M. Prevalence of anxiety and its correlates among older adults in Latin America, India and China: cross-cultural study. Br J Psychiatry 2011;199:485-491.

32. Copeland JRM, Beekman ATF, BraAm AW et al. Depression among older people in Europe: the EURODEP studies. World Psychiatry 2004;3:45-49.

33. Schaub RT, Linden M, Copeland JR. A comparison of GMS-A/AGECAT, DSM-III-R for dementia and depression, including subthreshold depression (SD) results from the Berlin Aging Study (BASE). Int J Geriatr Psychiatry 2003;18:109-117.

34. Thiébaut ACM, Bénichou J. Choice of time-scale in Cox's model analysis of epidemiologic cohort data: a simulation study. Stat Med 2004;23:3803-3820.

35. Scrucca L, Santucci A, Aversa F. Competing risk analysis using R: an easy guide for clinicians. Bone Marrow Transplant 2007;40:381-387.

36. Fine JP, GRAY RJ. A proportional hazards model for the subdistribution of a competing risk. J Am Stat Assoc 1999;94:496-509.

37. Pintilie M. Competing risks: a practical perspective. Chichester: Wiley; 2006.

38. Putter H, Fiocco M, Geskus RB. Tutorial in biostatistics: competing risks and multi-state models. Stat Med 2007;26:2389-2430.

39. GRAY RJ. A class of K-sample tests for comparing the cumulative incidence of a competing risk. Ann Stat 1988;16:1141-1154.

40. SCHeIKe TH, ZhANG MJ. Flexible competing risks regression modeling and goodness-of-fit. Lifetime Data Anal 2008; 14:464-483.

41. Gooley TA, Leisenring W, Crowley J, Storer BE. Estimation of failure probabilities in the presence of competing risks: new representations of old estimators. Stat Med 1999;18:695-706.
42. Kochar SC, Lam KF, YIP PS. Generalized supremum tests for the equality of cause specific hazard rates. Lifetime Data Anal 2002;8:277-288.

43. Lobo A, López-Anton R, Santabárbara J et al. Incidence and lifetime risk of dementia and Alzheimer's disease in a southern European population. Acta Psychiatr Scand 2011;124:372-383.

44. Donovan NJ, Locascio JJ, Marshall GA et al. Longitudinal association of amyloid beta and anxious-depressive symptoms in cognitively normal older adults. Am J Psychiatry 2018; 175:530-537.

45. Forsell Y, Jorm AF, Winblad B. Variation in psychiatric and behavioural symptoms at different stages of dementia: data from physicians' examinations and informants' reports. Dementia 1993;4:282-286.

46. Vance DE, Roberson AJ, McGuinness TM, Fazeli PL. How neuroplasticity and cognitive reserve protect cognitive functioning. J Psychosoc Nurs Ment Heal Serv 2010;48:23-30.

47. Perna G, Iannone G, Alciati A, Caldirola D. Are anxiety disorders associated with accelerated aging? A focus on neuroprogression Neural Plast 2016;2016:8457612.

48. Cuello AC. Early and late CNS inflammation in Alzheimer's disease: two extremes of a continuum? Trends Pharmacol Sci 2017;38:956-966.

49. Salim S, Chugh G, Asghar M. Inflammation in anxiety. Adv Protein Chem Struct Biol 2012;88:1-25.

50. Kelfve S, Thorslund M, Lennartsson C. Sampling and non-response bias on health-outcomes in surveys of the oldest old. Eur J Ageing 2013;10:237-245.

51. Copeland JR, Prince M, Wilson KCM, Dewey ME, Payne J, Gurland B. The Geriatric Mental State Examination in the 21 st century. Int J Geriatr Psychiatry 2002;17:729-732.

52. Braam AW, Copeland JR, Delespaul PA et al. Depression, subthreshold depression and comorbid anxiety symptoms in older Europeans: results from the EURODEP concerted action. J Affect Disord 2014;155:266-272.

53. Miloyan B, Bulley A, Bandeen-Roche K, Eaton WW, Gonçalves-Bradley DC. Anxiety disorders and all-cause mortality: systematic review and meta-analysis. Soc Psychiatry Psychiatr Epidemiol 2016;51:1467-1475.

54. Gray SL, Dublin S, Yu O et al. Benzodiazepine use and risk of incident dementia or cognitive decline: prospective population based study. BMJ 2016;2:190.

55. Moraros J, Nwankwo C, Patten SB, Mousseau DD. The association of antidepressant drug usage with cognitive impairment or dementia, including Alzheimer disease: a systematic review and meta-analysis. Depress Anxiety 2017;34:217-226.

56. Mawanda F, Wallace RB, McCoy K, Abrams TE. PTSD, psychotropic medication use, and the risk of dementia among US veterans: a retrospective cohort study. J Am Geriatr Soc 2017;65:1043-1050. 